\title{
Análisis comparativo del Modelo de Apoyo Estudiantil en The Open University (Inglaterra), con el Modelo de Apoyo Estudiantil en la Universidad Estatal a Distancia (Costa Rica)
}

\author{
Tobías Brizuela-Gutiérrez* \\ (iD) https://orcid.org/0000-0001-7291-0634 \\ Elizabeth Cascante-Ramirez** \\ (iD) https://orcid.org/0000-0001-5990-5507 \\ Sergio Gómez-Atencio*** \\ iD https://orcid.org/0000-0002-8938-1422
}

Recibido: 24 de setiembre, 2018 - Aceptado: 18 de octubre, 2018

\section{Formato de citación según APA}

Brizuela-Gutiérrez, T., Cascante-Ramírez, E. y Gómez-Atencio, S. (2018). Análisis comparativo del Modelo de Apoyo Estudiantil en The Open University (Inglaterra), con el Modelo de Apoyo Estudiantil en la Universidad Estatal a Distancia (Costa Rica). Revista Espiga, 17 (36), 225-239. Doi: http://dx.doi.org/10.22458/re.v17i36.2176

\section{Formato de citación según Chicago-Deusto}

Brizuela-Gutiérrez, Tobías, Elizabeth Cascante-Ramírez y Sergio Gómez-Atencio. «Análisis comparativo del Modelo de Apoyo Estudiantil en The Open University (Inglaterra), con el Modelo de Apoyo Estudiantil en la Universidad Estatal a Distancia (Costa Rica)». Revista Espiga 17, n. ${ }^{\circ} 36$ (julio-diciembre, 2018): 225-239. Doi: http:// dx.doi.org/10.22458/re.v17i36.2176

* $\quad$ Bachiller en la Enseñanza del Inglés para I y II ciclos, Universidad Estatal a Distancia. Licenciatura en Educación con énfasis en Currículo y Evaluación, Universidad de las Ciencias y el Arte. Máster en Educación con énfasis en la Enseñanza del Inglés, Universidad Latina de Costa Rica. Profesor titular del área de Enseñanza del Inglés y encargado de la Cátedra de Enseñanza del Inglés y docente de grado de asignaturas relacionadas con la carrera de Enseñanza del Inglés para I y II ciclos, UNED, Costa Rica. Correo: tbrizuela@uned.ac.cr

** Bachiller en Inglés y licenciada en Lengua Inglesa, Universidad de Costa Rica. Máster en Psicopedagogía, Universidad Latina de Costa Rica. Tutora de la carrera de Enseñanza del Inglés para I y II ciclos y encargada de la Cátedra de Lengua Inglesa de la Universidad Estatal a Distancia, Costa Rica. Correo: ecascante@uned.ac.cr

*** Bachiller en Inglés, Universidad de Costa Rica. Licenciado en Docencia, Universidad San Marcos. Máster en la Enseñanza del Inglés como Lengua Extranjera, Universidad de Costa Rica. Tutor de la Cátedra de Lengua Inglesa de la carrera de Enseñanza del Inglés para I y II ciclos, UNED. Profesor de los cursos Inglés 100\% en Línea del Centro de Idiomas, UNED, Costa Rica. Correo: sgomez@uned.ac.cr 
Para que los estudiantes de un sistema de educación a distancia lleven a cabo sus estudios exitosamente, es necesario contar con un modelo definido de apoyo estudiantil. The Open University cuenta con un sistema tal que ha ido afinando desde sus inicios. Si bien existen diferencias en cuanto al tipo de población que atienden The Open University y la UNED, así como en las estructuras de ambas universidades, la UNED puede incorporar elementos del modelo de apoyo estudiantil de esta institución.

El presente artículo desarrolla un corto análisis comparativo entre los modelos de ambas instituciones con el fin de identificar qué aspectos se podrían adoptar en la carrera de Enseñanza del Inglés para I y II ciclos que se imparte en la UNED.

Palabras clave: Modelo de apoyo estudiantil, educación a distancia, cognitivo, afectivo, sistémico.

\section{Introducción}

Estudiar en un modelo a distancia requiere que el estudiante cuente con una serie de habilidades que, en algunos casos, va más allá de lo que un sistema de enseñanza tradicional procura ${ }^{1}$. Además de las habilidades cognitivas, sociales, organizacionales y tecnológicas, existen factores que toda institución de educación superior le exige a los estudiantes y con los que deben lidiar. Con un panorama tal, no es de extrañar que cursar una carrera en una institución a distancia represente un reto que, desafortunadamente, muchas veces termina en deserción o en un proceso que se extiende más de lo necesario mientras los estudiantes aprenden a navegar por este mar de variables ${ }^{2}$.

Lo anterior, lleva a las instituciones de educación superior a distancia a plantearse la necesidad de darle al estudiante suficiente apoyo para que pueda cumplir con el reto que se planteó. Esto, sobre todo, si se parte de la premisa de que el estudiante es el centro del proceso de enseñanza, no solo en la educación a distancia, sino también de la educación en general ${ }^{3}$.

El concepto de apoyo estudiantil (Student Support) se ha ido desarrollando a través de los años y está, en el presente, bastante consolidado ${ }^{4}$. The Open University, ubicada en Inglaterra y establecida desde 1969, cuenta con un sistema de apoyo estudiantil teóricamente fundamentado y llevado a la práctica desde hace varios años ${ }^{5}$. Esta universidad atiende a personas de varios lugares del mundo, con un total de 174739 estudiantes según datos del 2016, de los cuales 7430 provienen del exterior del Reino Unido. La relación entre hombres y mujeres en esta población estudiantil es de 40:60 para el nivel de pregrado y de 53:47 para el nivel de posgrado. En el nivel de pregrado la mayoría de estudiantes se

1. Johnny Cartín Quesada, «Los modelos pedagógicos centrados en el estudiante: apuntes sobre los procesos de aprendizaje y enseñanza» (Programa de investigación en fundamentos de educación a distancia, Vicerrectoría de investigación, Universidad Estatal a Distancia, 2012).

2. Ibíd.

3. Ibíd.

4. Tait, Alan, «On Institutional Models and Concepts of Student Support Services: The Case of the Open University» (UK, 2018).

5. Ibíd. 
ubica entre los 25 y 34 años (34\%), y en el nivel de posgrado entre 35 y 44 años (34\%) 6 . Es importante acotar que The Open University, como lo dice su nombre, responde al modelo de educación abierta, por lo tanto no establece el egresarse del sistema de educación secundaria como un requisito de ingreso a la universidad.

The Open University ha manifestado que tiene una visión del aprendizaje en línea que es personalizada, la cual permite que el aprendizaje sea cualquier cosa menos distante. Ellos aseguran que su método provee un aprendizaje abierto y acompañado (Open and Supported). Esta idea de abierto permite que los estudiantes escojan sus cursos y planifiquen sus estudios a su ritmo personal y desde cualquier lugar que deseen.

Estas características de flexibilidad y accesibilidad fundamentan la idea de «Open». En relación con la idea de acompañado (Supported), The Open University asegura que «desde el día uno y durante todo el curso de los estudios, el estudiante tendrá el acompañamiento de un tutor designado, otros actores del personal administrativo, los compañeros de los cursos, los miembros de la facultad y la amplia comunidad estudiantil universitaria con los cuales se pueden conectar por una variedad de medios» ${ }^{7}$.

El sistema Open and Supported puede servir de modelo para la Universidad Estatal a Distancia (UNED), ya que ambas instituciones comparten varias similitudes en lo que a su misión y a su visión se refiere. Por esta razón, en el siguiente artículo se pretende analizar el modelo de The Open University, compararlo con el modelo de apoyo estudiantil de la UNED y determinar si este último se puede beneficiar de la experiencia y enriquecerse con el conocimiento de dicha institución. Todo esto con el objetivo de fortalecer los recursos administrativos al servicio de los procesos pedagógicos, específicamente de la carrera de Enseñanza del Inglés para I y II ciclos.

\section{Fundamentación teórica de un modelo de apoyo estudiantil}

The Open University va un paso más allá de considerar al estudiante como el centro de la educación, esto al enfatizar el servicio al estudiante en su labor académica ${ }^{8}$. Es necesario primero definir y delimitar el concepto de apoyo estudiantil para la educación a distancia. Este concepto se refiere a «la variedad de servicios tanto para individuos como para estudiantes que son parte de un grupo que complementan los materiales del curso o los recursos de aprendizaje. Los mismos son uniformes para todos los estudiantes y se perciben como lo más importante que ofrecen las instituciones de educación abierta o a distancia. De tal manera, el apoyo estudiantil se distingue de la totalidad de actividades de un programa de educación abierta o a distancia. Este, en general, se puede decir que tiene como objetivo principal el apoyar a los estudiantes» ${ }^{9}$.

\footnotetext{
6. The Open University, Facts and Figures 2015/16.

7. Tait, Alan, «Planning Student Support for Open and Distance Learning. Open Learning: The Journal of Open and Distance Learning» (2000).

8. Ibíd.

9. Ibíd.
} 
De acuerdo con el profesor Alan Tait, el apoyo estudiantil tiene tres funciones principales:

1. Cognitiva: apoya y desarrolla el aprendizaje a través de la mediación entre los estudiantes individuales y los materiales de curso y los recursos de aprendizaje estándar y uniformes.

2. Afectiva: provee un ambiente de apoyo para los estudiantes, que crea compromiso y promueve la autoestima.

3. Sistémica: establece procesos administrativos y sistemas de administración de información que son efectivos, transparentes, y amigables con el estudiante.

\section{Modelo de apoyo dirigido al factor cognitivo}

Está de más apuntar la importancia del factor cognitivo en un proceso de enseñanzaaprendizaje. Dado este hecho, cualquier modelo de apoyo estudiantil debe incluir este aspecto. Como ya se mencionó, la función cognitiva de un sistema de apoyo estudiantil es ayudar y desarrollar el aprendizaje a través de la mediación entre los estudiantes individuales y los materiales de curso y los recursos de aprendizaje estándar y uniformes ${ }^{10}$.

El concepto de mediación se puede definir como «aquellos esfuerzos pedagógicos y didácticos vinculados a la ayuda que la institución o docente puedan ofrecer para que el estudiante logre gestionar y efectuar con éxito su proceso de formación $\rangle^{11}$. La educación a distancia es una modalidad más mediada que las modalidades presenciales, dado que en estas últimas el docente es quién carga con el mayor peso del proceso de enseñanza al ser este quien interactúa constantemente con los estudiantes. Sin embargo, en un modelo a distancia, es la institución la que comparte gran parte de esta carga con el docente ${ }^{12}$.

Es necesario que la institución tenga medios de interacción con el estudiante que se dirijan a «la construcción de los diferentes saberes profesionales de la universidad y hacia la creación de competencias críticas y analíticas en los sujetos del aprendizaje» ${ }^{13}$. De manera que es fácil concluir que el éxito del estudiantado depende en gran medida de lo que la institución o el docente le faciliten a este.

Precisa definir la función cognitiva un poco más para determinar qué tipo de servicios se le deberían ofrecer al estudiantado con el fin de cubrir esta área. De acuerdo con el modelo pedagógico de la UNED, el estudiante es el centro del proceso de enseñanzaaprendizaje y se espera que el aprendizaje sea auto-dirigido, autónomo y auto-regulado. El estudiante es el «responsable y gestor de su aprendizaje» ${ }^{14}$. Esta situación implica que

\footnotetext{
10. Cartín, Los modelos pedagógicos...

11. Ibíd.

12. Ibíd.

13. Ibíd.

14. Ibíd.
} 
el estudiante debe contar con un abanico de habilidades para tener éxito en sus estudios. Entre estas habilidades se incluyen las siguientes:

- $\quad$ Estrategias de estudio.

- Organización del tiempo.

- Identificación de las propias necesidades de aprendizaje.

- Desarrollo de habilidades para la adquisición continua de nuevos conocimientos.

El concepto de aprendizaje auto-dirigido está muy relacionado con el enfoque constructivista, el cual en términos generales empapa el modelo pedagógico de la UNED ${ }^{15}$. Hablar de un enfoque constructivista unificado es difícil, pero se puede mencionar una serie de principios generales que se diría responden a una filosofía constructivista. Para Cartín ${ }^{16}$ : «en general, podemos considerar como constructivista todo modelo pedagógico que conciba el aprendizaje como un proceso de construcción (y no solo de transferencia o asimilación asociativa) de conocimiento».

Este movimiento postula cuatro estrategias principales para que el aprendizaje se dé exitosamente y para que la capacidad cognitiva se vea fortalecida ${ }^{17}$ :

- Confrontar el nuevo concepto con otros afines.

- Basar el nuevo aprendizaje en el conocimiento previo (ideas y esquemas) del alumno.

- Aplicar el concepto enseñado a situaciones concretas, con el fin de que el estudiante amplíe su significado.

- Prever el cambio conceptual y la reelaboración de la estructura mental a partir de la incorporación del nuevo concepto.

Dichas estrategias, además de beneficiar el aprendizaje del estudiante, le ayuda a aprender a aprender. Todos los procesos anteriores apuntan a que es totalmente necesario que el estudiante reciba apoyo suficiente de la institución de manera que pueda cumplir con el gran reto que representa estudiar en una universidad a distancia. Este apoyo puede tomar diferentes formas, muchas de las cuales son ofrecidas por el modelo de apoyo estudiantil de The Open University.

15. Ibíd.

16. Ibíd.

17. Ibíd. 
Con el fin de contextualizar el factor afectivo en la educación abierta o a distancia, es importante referirse a la motivación dentro de los procesos de enseñanza-aprendizaje. Esto por cuanto la motivación, según la define Ajello, citado por María Luisa Naranjo ${ }^{18}$, «debe ser entendida como la trama que sostiene el desarrollo de aquellas actividades que son significativas para la persona y en las que esta toma parte. En el plano educativo, la motivación debe ser considerada como la disposición positiva para aprender y continuar haciéndolo de una forma autónoma». Esta disposición positiva es a lo que Trechera ${ }^{19}$ se refiere con «aquello que moviliza a la persona para ejecutar una actividad», o sea, este es el «proceso por el cual el sujeto se plantea un objetivo, utiliza los recursos adecuados y mantiene una determinada conducta, con el propósito de lograr una meta».

La motivación es, por lo tanto, una de los pilares del factor emocional. Pintrich y De Groot $^{20}$ enfatizan el rol de la motivación en ambientes educativos al exponer lo que ellos consideran tres categorías de principal relevancia:

1. Componente de expectativas: se refiere a las creencias acerca de la propia capacidad para ejecutar una tarea.

2. Componente de valor: se asocia a las metas y percepciones sobre la importancia e interés de dicha tarea.

3. Componente afectivo: hace referencia a las consecuencias afectivo-emocionales que generan la realización de una tarea, así como de los resultados de éxito o fracaso académico.

Por consiguiente, Pintrich y De Groot concluyen que «la persona se motiva más por el proceso de aprendizaje cuando confía en sus capacidades y posee altas expectativas de auto-eficacia, además de valorar las actividades educativas y responsabilizarse de los objetivos de aprendizaje».

Dado lo anterior y ante la naturaleza - para muchos aprendientes desconocida-y las exigencias de un emergente modelo educativo abierto, se hace necesario un nuevo abordaje del factor emocional. Esto por cuanto el impacto de las emociones en el éxito o fracaso académico es significativo ${ }^{21}$. El cambio de un modelo presencial-tradicional a uno abierto o a distancia provoca en el estudiantado una respuesta emocional, la cual afecta la experiencia del aprendizaje en línea ${ }^{22}$. Es, por tanto, inadmisible pensar en un modelo

18. María Luisa Naranjo Pereira, «Motivación: perspectivas teóricas y algunas consideraciones de su importancia en el ámbito educativo», Revista Educación, 33(2), (2009): 153-170.

19. Ibíd, 154.

20. Paul R. Pintrich y Elisabeth V. De Groot, «Motivational and Self-Regulated Learning Components of Classroom Academic Performance», Journal of Educational Psychology, Vol. 82, 1, (1990): 33-40.

21. Mariana Hentea, «A Perspective on Fulfilling the Expectations of Distance Education», Proceedings of the 4th conference on Information technology curriculum.

22. Kalogiannakis Michail, «Emotions Experienced by Learners and their Development through Communication with the Tutor-Counsellor», European Journal of Open, Distance and e-Learning, Vol. 18, n. ${ }^{\circ} 2(2015)$ 
educativo innovador que obvie este factor. El no establecer esta referencia conllevaría a asumir que la comunidad estudiantil es un conglomerado de individuos sin emociones y se estaría desaprovechando la influencia de lo emotivo en el proceso educativo, según lo exponen Brookfield, Lehman y Lipman ${ }^{23}$.

Martha Cleveland-Innes y Prisca Campbel1 ${ }^{24}$ explican que es necesario considerar que la tecnología ha venido a facilitar los procesos de aprendizaje en la educación a distancia; no obstante, los requerimientos tecnológicos necesarios para sobrellevar las demandas de un modelo educativo abierto de este tipo, acarrean inevitablemente el surgimiento y el desarrollo de nuevas habilidades. Estas habilidades, que por su parte desencadenan respuestas emocionales, tanto positivas como negativas, repercuten como un impacto tangible en los procesos de aprendizaje. De ahí la importancia de que las instituciones educativas doten de recursos a los programas de apoyo enfocados en lo afectivo.

Lo anterior, en un principio, podrá parecer poco concreto; sin embargo, se puede materializar fácilmente a través de los ya existentes apoyos sistémicos (plataformas, componentes en línea y sistemas de administración de información efectivos, para mencionar algunos) e indudablemente por medio de la red de apoyos cognitivos. Es decir, no se puede desasociar los factores porque los tres son inherentes a la experiencia de aprendizaje. De tal manera que la relación afectivo-cognitivo-sistémico debe considerarse como un todo en lo que a educación a distancia se refiere.

La relación entre emoción y aprendizaje, de acuerdo con Cleveland-Innes y Campbell, no había recibido la atención que merece hasta inicios de los 2000. Esto se debe a que emoción y aprendizaje se habían considerado elementos antagónicos. No fue hasta el 2002 que Bloom introdujo la noción de lo afectivo como parte del campo del aprendi$\mathrm{zaje}^{25}$. El ignorar las emociones como parte de la respuesta humana a eventos internos y externos, aducen LeDoux, Plutchick, Stets, Turner, Wosnitza y Volet, implica menospreciar un elemento nuclear de la experiencia humana ${ }^{26}$.

Dado lo anterior, es de especial importancia analizar esta correlación en la práctica. Los programas de educación abierta o a distancia deben abocarse a un modelo integrado de apoyo a los estudiantes que comprenda elementos como autoestima, confianza y perseverancia, entre otros. Este modelo es precisamente el que ha implementado The Open University con mucho éxito.

The Open University, en su afán de democratizar y universalizar la educación, ha creado apoyos que van desde lo socioeconómico y cognitivo hasta lo afectivo. En lo que a lo afectivo se refiere, este apoyo se estructura por medio de una red en la cual están los siguientes elementos:

- Stress Buster (Destructor de estrés): consiste en una serie de pasos para guiar al estudiante hacia una vida libre de estrés.

23. Martha Cleveland-Innes y Prisca Campbell, «Emotional Presence, Learning, and the Online Learning Environment», The International Review of Research in Open and Distance Learning.

24. Ibíd.

25. Ibíd.

26. Michalinos Zembylasa, «The Role of Emotions in the Experience of Online Learning: Challenges and Opportunities», Educational Media International, Vol. 45, n. ${ }^{\circ}$ 2, (June 2008): 107-117. 
- Plataforma anti-acoso en línea: pretende detectar y erradicar las diferentes formas de abuso en línea que pueden presentarse en las plataformas de aprendizaje con que cuenta The Open University.

- OU Analyse: detección de estudiantes en riesgo de deserción por medio de un sistema computarizado. Este método facilita la identificación e intervención para lograr la retención de estudiantes.

- SST (Student Support Teams): explora el comportamiento de los estudiantes y ayuda a identificar aquellos que podrían necesitar una intervención.

Todo lo anterior, aunado a los apoyos cognitivos y sistémicos, han hecho de The Open University un modelo exitoso capaz de atender una población aproximada de 174 839 estudiantes distribuidos en 141 países. Las particularidades e idiosincrasias de sus diferentes públicos meta hacen necesaria la creación de una red de apoyos tan variopinta como su población de estudiantes.

\section{Modelo de apoyo dirigido al factor sistémico}

El sistema de estudio a distancia plantea retos específicos a los estudiantes en relación con sus requerimientos y sus demandas académicas. De manera que es imperante tener un sistema de apoyo al estudiantado que les ayude a cumplir todas las exigencias académicas con éxito.

Una de las vertientes esenciales del modelo de ayuda a los estudiantes de The Open University, es el relacionado con la parte sistémica. The Open University tiene muy bien claro en su filosofía educativa que la satisfacción de sus estudiantes ha sido una de las claves para posicionarlos como una de las mejores universidades de educación a distancia en el Reino Unido. El autor y profesor de The Open University, Alan Tait, en su artículo «Reflections on Student Support in Open and Distance Learning», establece claramente que el apoyo al estudiante -especialmente la guía, la orientación, la información eficiente y los sistemas administrativos-impactan no solo el proceso de enseñanza y aprendizaje, sino que contribuyen a la parte afectiva, pues refuerza el sentido de confianza, de autoestima y de progreso ${ }^{27}$.

Para Tait, la parte sistémica del modelo de The Open University (la relacionada con las reglas) tiene como fin último la permanencia del estudiantado en sus estudios, es decir, evitar la deserción. En el caso de The Open University, la institución se esfuerza por tener índices de deserción en porcentajes mínimos. Tait, refiriéndose a las funciones primordiales de los sistemas de apoyo estudiantil, indica que en la parte sistémica se deben establecer procesos administrativos y mecanismos gerenciales de información que sean efectivos, transparentes y amigables para los beneficiarios.

La necesidad de establecer mecanismos de apoyo sistémico a los estudiantes que son parte de la educación superior y a distancia, ha sido una gran preocupación para

27. Alan Tait, International Review of Research in Open and Distance Learning, Volume 4, Number 1 (2003). 
las autoridades de las instituciones educativas del mundo. La autora Jane Briendly ${ }^{28}$ comenta acerca de las dificultades en relación con los servicios ofrecidos a los estudiantes de la educación a distancia: En los primeros años de la educación a distancia el enfoque educacional se centraba en el acceso y la disponibilidad de las opciones de aprendizaje a expensas de la experiencia individual y particular del aprendiz. Tal visión se vio permeada con altos márgenes de deserción ${ }^{29}$. Consecuentemente, la idea de sistemas de apoyo al estudiantado fue en primer plano concebida con la intención de minimizar estos porcentajes altos de «bajas en el sistema».

Briendly lo puntualiza de forma clara ${ }^{30}:$ «proveer servicios de apoyo al estudiantado es una de las claves de todos aquellos que están comprometidos con posicionar al estudiantado en el centro de todo el proceso, de modo que se pueda ser más competitivo y estar mejor catalogado frente a la demanda de educación accesible y de alta calidad».

\section{Servicios que se deben ofrecer en un sistema de apoyo al estudiante}

Según lo expone Tait, un sistema de apoyo al estudiante debería incluir los siguientes servicios $^{31}$ :

- Tutorías.

- Servicios de biblioteca.

- Residencias estudiantiles.

- Servicios de guía y consejería.

- Centros de estudio y de evaluación.

- Diferentes sistemas de administración de información.

- Servicios de consulta, admisión y consejería pre-estudio.

- Evaluación de conocimientos previos y reconocimiento de créditos.

- Enseñanza individualizada, incluyendo en algunos casos evaluación continua.

- Materiales que ayuden al desarrollo de habilidades de estudio, planeamiento de un programa de estudio o desarrollo profesional.

- Servicios diferenciados para estudiantes con necesidades especiales (por ejemplo, discapacidades, habitación en zonas alejadas, privados de libertad).

\section{Servicios que ofrece la UNED dentro de su sistema de apoyo estudiantil}

El documento Modelo Pedagógico de la UNED fue aprobado por el Consejo Universitario en la sesión n. ${ }^{\circ}$ 1714, artículo IV, inciso 3, del 9 de julio del 2004. Este establece que se decidió fundar una universidad estatal que trabajase con modalidad a distancia, de acuerdo con los modelos de la UNED de España y The Open University ${ }^{32}$. En dicho

\footnotetext{
28. Jane Briendly, Learners and Learner Services: The Key to the Future in Open Distance Learning (1995).

29. Ibíd.

30. Ibíd.

31. Tait, Planning Student Support..., 287-299.

32. Modelo pedagógico, UNED (febrero 2005): 3.
} 
modelo, se plantean lineamientos institucionales diferentes de los que, para efectos de este artículo, se puntualizan los siguientes dos:

1. Orientar, con criterio pedagógico, el uso de una amplia gama de medios y apoyos didácticos, incorporando las nuevas alternativas de comunicación por medios telemáticos cuando sea pertinente y se constate el acceso de los estudiantes a estos medios.

2. Trascender lo meramente didáctico, de manera que se posibilite la formación integral y permanente, la socialización y el desenvolvimiento pleno de las potencialidades de cada persona, así como una ética personal y compromiso social.

La UNED también posee una estructura muy bien definida en relación con los apoyos al estudiantado. La Vicerrectoría Ejecutiva, en su espacio dentro de la intranet, visibiliza una de sus dependencias claves en relación con el apoyo al estudiantado: la Dirección de Asuntos Estudiantiles (DAES) ${ }^{33}$. Esta dependencia tiene una meta muy bien definida, cuyo fin es desarrollar una serie de acciones y programas propios atinentes a la promoción de la vida estudiantil. Todas estas acciones buscan potenciar el desenvolvimiento de las habilidades de los estudiantes de todo el país. El estudiante se enmarca en una visión humanística e integral tendientes a la permanencia y éxito en su formación.

La visión de la DAES, con respecto a los factores cognitivo-afectivo-sistémico, es contundente:

«El Área de Vida Estudiantil de la UNED es la instancia responsable de liderar y fortalecer estrategias y procesos institucionales, para la formación y el desarrollo integral de la población estudiantil bajo una visión humanista y holística que promueva su acceso al sistema, su incorporación, su adaptación, su permanencia y su logro académico en la Universidad» ${ }^{34}$.

Cuatro pilares esenciales integran la estructura organizacional de la DAES:

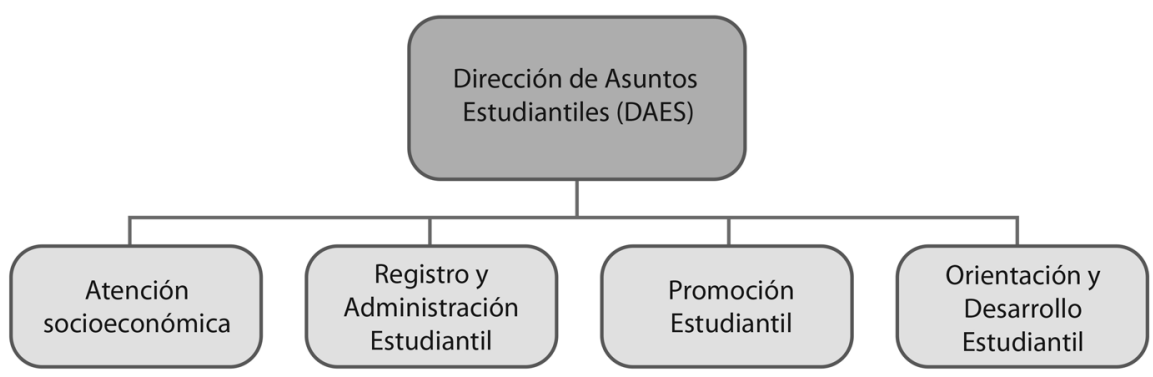

Fuente: Página principal de la UNED, Vicerrectoría Ejecutiva.

33. Sitio web de la UNED. Acceso: 01 de octubre de 2018, https://www.uned.ac.cr/vidaestudiantil/ quienes-somos/93-direccion-de-asuntos-estudiantiles

34. Ibíd. 
La UNED, por medio de las acciones que ejecutan cada una de las dependencias anteriores, se ha planteado metas claras en relación con el apoyo que se les puede brindar a los estudiantes en todas las áreas posibles, de modo que se garantice la permanencia de los estudiantes en el sistema. Es interesante ver dos de las dependencias y las áreas que estas pretenden cubrir.
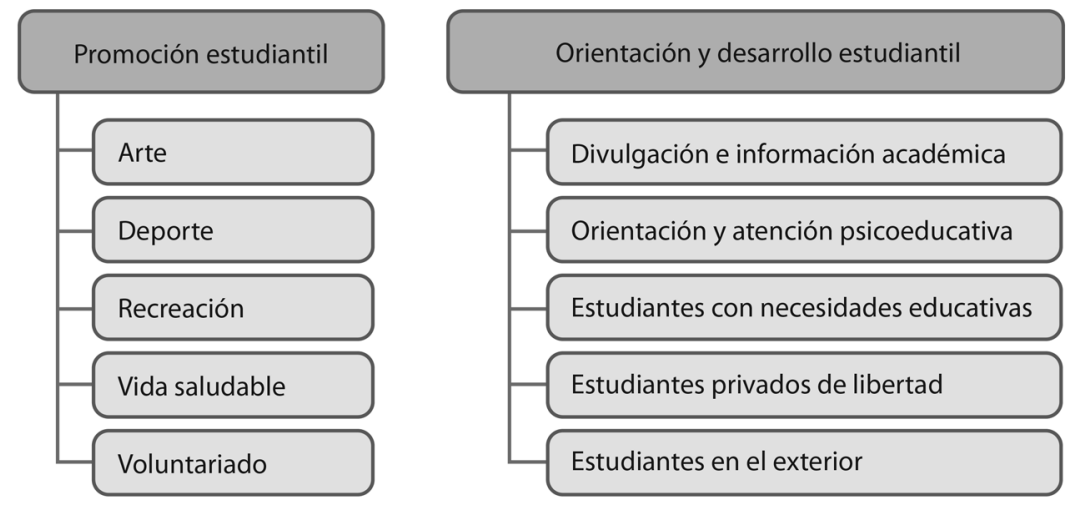

Fuente: Página principal de la UNED, Vicerrectoría Ejecutiva.

Sin duda, la visión y misión de la UNED se orientan a una postura holística de la educación que visualiza al estudiante como un ser integral y al cual hay que ayudar en todas las áreas de su ser. Aun así, se siguen planteando retos en relación con los índices de permanencia y deserción del estudiantado de la UNED.

\section{CUADRO 1}

Comparativo de servicios que ofrecen ambas universidades

\begin{tabular}{|c|c|c|}
\hline Criterio de comparación & The Open University & UNED \\
\hline $\begin{array}{l}\text { Un modelo de aprendizaje } \\
\text { integral y apoyo estudiantil } \\
\text { (Model for Integrated Learning } \\
\text { and Learner Support, MILLS) }\end{array}$ & $\begin{array}{l}\text { El modelo tiene estas características: } \\
\text { - Toma en cuenta las características de las } \\
\text { materias que está llevando el estudiante. } \\
\text { - Incluye apoyos de aprendizaje. } \\
\text { - Integran los Servicios Académicos y } \\
\text { las Escuelas (Academic Services and } \\
\text { Faculties). } \\
\text { - Brinda un apoyo uniforme con canales } \\
\text { claros de comunicación. } \\
\text { - Apoya la retención, progreso y } \\
\text { compleción. }\end{array}$ & $\begin{array}{l}\text { Cuenta con varios servicios, como la Dirección } \\
\text { de Asuntos Estudiantiles (DAES), asistido } \\
\text { por el Programa de Aprendizaje en Línea } \\
\text { (PAL), el Programa de Producción Electrónica } \\
\text { Multimedial (PEM) y el Centro de Información, } \\
\text { Documentación y Recursos Bibliográficos } \\
\text { (CIDREB). Sin embargo, la diferencia radica } \\
\text { en la sistematización y articulación de dichos } \\
\text { elementos. }\end{array}$ \\
\hline
\end{tabular}


CUADRO 1 (Continuación)

\section{Criterio de comparación}

Intervenciones universales

(Universal Interventions) del modelo MILLS

\section{The Open University}

Estas intervenciones se dan en diferentes momentos y tienen la característica de ser proactivas. Los siguientes pueden ser momentos en los que se dan:

- Durante la preparación para el módulo (curso).

- Durante los estudios de certificación.

- Durante la preparación de los estudios

- Mientras se brinda seguimiento cuando un estudiante no realiza una prueba $u$ obtiene una calificación muy baja.

Cuando un estudiante no entrega una tarea.

- Al planificar unas semanas cargadas de trabajos.

- Preparándose para los exámenes.

- Preparándose para entregar el trabajo final.

- Al planificar los próximos pasos para alcanzar la meta de estudios.

- Preparándose para exámenes de reposición u otras evaluaciones. para asegurar el éxito de los
Sistema de análisis de datos estudiantes (Analytics)

Incluye un sistema para registrar datos de aprobación y deserción, entre otros, y el análisis de dichos datos. El sistema $O U$ Analyse permite detectar patrones en el comportamiento de los estudiantes en las plataformas virtuales que representen un indicador de la necesidad de una intervención institucional (académica, psicológica, emocional, social, pedagógica).

Un programa para llevar un registro del estudiante, llamado VOICE
El sistema lleva registro del récord académico y contactos anteriores con el personal de apoyo al estudiante (learner support staff). Provee un resumen de la comunicación con el estudiante.

\section{UNED}

Se cuenta con orientaciones académicas que se ponen a disposición en la web un mes antes de la matrícula. Además, existen apoyos curriculares a estudiantes con necesidades especiales. Esto es coordinado por una de las dependencias de la DAES. La carrera de la Enseñanza del Inglés, a través de iniciativas de las cátedras, ha promovido planes estratégicos de mejoramiento académico (PEMA), plan de nivelación lingüístico, prácticas extra, materiales multimediales complementarios y exámenes de reposición, entre otros mecanismos tendientes al acompañamiento integral del estudiantado.
Incluye un sistema de registro de datos en el departamento de Registro, que se vincula al entorno estudiantil.
La UNED no posee un sistema integrado o sistematizado que brinde apoyo al estudiante a través de una herramienta como VOICE. Sin embargo, existen algunos mecanismos con la finalidad de apoyar al estudiante. Por ejemplo, los centros universitarios cuentan con profesionales orientadores, así como el PADD (Programa de Apoyo Didáctico a Distancia), cuya finalidad es ofrecer a los estudiantes la posibilidad de realizar sus consultas académicas por diferentes vías de comunicación (teléfono, fax, correo electrónico y mensaje de voz). 
CUADRO 1 (Continuación)

Criterio de comparación

Página de internet con información variada

\section{The Open University}

Por ejemplo, una «Guía de supervivencia» que ayuda al estudiante a navegar en la OU.

\section{UNED}

Cuenta con una página web y un canal en YouTube con una serie de recursos audiovisuales que apoyan al estudiante al momento de incorporarse a la institución. También, hay varias redes sociales enfocadas en darle apoyo al estudiante.

Un equipo de apoyo al estudiante conformado y definido, que incluye personal académico, tutores y demás elementos de apoyo estudiantil

El personal de apoyo estudiantil está capacitado para manejar una gama amplia de asuntos que los estudiantes pueden encontrar y, de esta manera, ayudar a identificar soluciones. hace el encargado de cátedra y los asistentes
En su mayoría, muchas de estas funciones las académicos y administrativos. Sin embargo, la UNED fomenta la autorregulación del estudiantado, es decir, que en la medida de lo posible puedan encontrar las respuestas en los mecanismos provistos para estos efectos. En este caso, se debe señalar la diferencia en la visión del estudiante que ambas instituciones tienen.

\section{Conclusiones y recomendaciones}

No hay duda de que la educación a distancia plantea un gran reto a las instituciones que deciden fundamentar sus prácticas en dicho modelo. The Open University, la Universidad Nacional de Educación a Distancia de España y la Universidad de Athabasca de Canadá, son claros ejemplos de sistemas abiertos y a distancia que han podido sobrevivir a través de los tiempos y las dificultades. Esto sin perder de vista que el centro de todos sus procesos son los estudiantes. De ahí que dichas universidades invierten mucho de su presupuesto en idear y perfeccionar sistemas de apoyo al estudiante que permiten a estos navegar, subsistir y completar con éxito sus metas académicas.

La UNED, fundada en 1977 y con un modelo basado en la experiencia de las universidades mencionadas anteriormente, ha procurado también orientar todos sus procesos al proveer mecanismos y dependencias en la universidad que permitan a sus estudiantes ser también exitosos en el alcance de sus metas y propósitos académicos. No obstante, a partir de la experiencia vivida en The Open University y al confrontar su modelo de apoyo al estudiante con el de la UNED, surgen todavía algunos retos en este sentido y, en particular, para la carrera de la Enseñanza del Inglés para I y II ciclos, programa para el cual laboran los autores de este artículo. Los retos detectados son:

1. Crear un protocolo de intervención estudiantil dentro del programa y las cátedras con el fin de determinar acciones concretas por seguir en casos específicos. Así mismo, y como parte de este protocolo, se podrían tipificar situaciones que ameritan intervenciones y coordinarse con las personas o dependencias involucradas.

2. Capacitar a los tutores y encargados de cátedra en el uso e importancia de dicho protocolo.

3. Crear un sistema informático o digitalizado que facilite llevar un control y seguimiento de las intervenciones y ayudas que se le brindan a los estudiantes. 
4. Tomando como ejemplo el sistema VOICE, diseñar un mecanismo que pueda vincular las dependencias de la UNED que dan apoyo al estudiante, así como a los profesionales que participan de estos procesos. Esto con la finalidad de brindar un servicio integral y articulado.

5. Maximizar el recurso técnico y crear plataformas de contención y prevención.

6. Propiciar espacios de reflexión al final de cada cuatrimestre o en el Taller Valorando la Carrera, con el fin de evaluar el desempeño de los estudiantes e identificar algunos que puedan presentar condiciones particulares y que pudieran llevar a un posible abandono de los estudios. Esto permitiría no solamente fomentar una cultura sistematizada de apoyo al estudiante, sino que ayudaría a aumentar los porcentajes de retención y permanencia en los estudios universitarios.

\section{ABSTRACT}

Comparative Analysis of the Student Support Model of The Open University, the United Kingdom, and the Student Support Model of the Distance State University

\section{(Universidad Estatal a Distancia), Costa Rica}

In order for students in a distance learning setting to be successful, it is necessary to have a defined model of student support. The Open University (OU) has such a model, which has been fine-tuned throughout its existence. In spite of the differences between the OU and the Distance State University (UNED) in terms of the population they cater to and their structure, the UNED can certainly adopt elements from the OU's model.

This article presents a short comparative analysis between both universities' models so as to identify which aspects could be adopted by UNED.

Key words: Student support model, distance education, cognitive, affective, systemic.

RÉSUMÉ

Analyse comparative des modèles de soutien aux étudiants de l'Open University en Angleterre et de l'Universidad Estatal a Distancia au Costa Rica

Le système de soutien aux étudiants est essentiel dans l'enseignement à distance pour qu'ils réussissent leurs études. L'Open University possède un modèle quasi unique qui a affiné depuis sa création. Bien qu'il y ait de différences entre le type de public qu'accueillent les deux universités et ainsi que dans leurs structures, l'UNED (Université de l'État à Distance) peut emprunter des éléments du modèle de soutien aux étudiants de l'Open University.

Cet article développe une petite analyse comparative entre les deux modèles afin d'identifier des aspects qui puissent être adoptés par la filière de l'Enseignement de l'anglais à l'école primaire de l'UNED.

Mots-clés: Soutien aux étudiants, enseignement à distance, cognition, affectivité, systémique. 


\section{Bibliografía}

Briendly, Jane. «Learners and Learner Services: The Key to the Future in Open Distance Learning». The Open University, 1995.

Cartín Quesada, Johnny. «Los modelos pedagógicos centrados en el estudiante: apuntes sobre los procesos de aprendizaje y enseñanza». Programa de investigación en fundamentos de educación a distancia, Vicerrectoría de Investigación, Universidad Estatal a Distancia, 2012.

Cleveland-Innes, Martha y Prisca Campbell. «Emotional Presence, Learning, and the Online Learning Environment». The International Review of Research in Open and Distance Learning. Vol. 13, n. ${ }^{\circ} 4$, Research Articles. Octubre 2012

Hentea, Mariana, Mary Jo Shea y Lisa Pennington. «A Perspective on Fulfilling the Expectations of Distance Education». Proceedings of the $4^{\text {th }}$ conference on Information technology curriculum, CITC4'03, 2003, Lafayette, Indiana, USA. Octubre 16-18.

Kalogiannakis, Michail. «Emotions Experienced by Learners and their Development through Communication with the Tutor-Counsellor». European Journal of Open, Distance and e-Learning. Vol. 18, n. ${ }^{\circ}$ 2, 2015.

Naranjo Pereira, María Luisa. «Motivación: perspectivas teóricas y algunas consideraciones de su importancia en el ámbito educativo». Revista Educación 33(2), 2009: 153-170.

Pintrich, Paul R. y Elisabeth V. De Groot. «Motivational and Self-Regulated Learning Components of Classroom Academic Performance». Journal of Educational Psychology, Vol. 82, n. ${ }^{\circ} 1$ (1990): 33-40.

Tait, Alan. International Review of Research in Open and Distance Learning. Volume 4, Number 1 (2003).

Tait, Alan. «Planning Student Support for Open and Distance Learning». Open Learning: The Journal of Open, Distance and e-Learning. Volume 15, Issue 3 (2000).

Tait, Alan. «On Institutional Models and Concepts of Student Support Services: The Case of the Open University UK». Learner Support in Open, Distance and Online Learning Environments. Volume 9 (2018): 284-293.

The Open University. Facts and Figures 2015/16.

Universidad Estatal a Distancia. Documento Modelo Pedagógico. Febrero, 2005.

Universidad Estatal a Distancia. Departamento de Asuntos Estudiantiles. Acceso: 01 de octubre de 2018. https://www.uned.ac.cr/vidaestudiantil/quienes-somos/93-direccion-de-asuntos-estudiantiles Zembylasa, Michalinos, «The Role of Emotions in the Experience of Online Learning: Challenges and Opportunities». Educational Media International, Vol. 45, n. ${ }^{\circ}$ (June 2008): 107-117. 